Ferrata Storti Foundation

\title{
Poor prognosis of chromosome 7 clonal aberrations in Philadelphia-negative metaphases and relevance of potential underlying myelodysplastic features in chronic myeloid leukemia
}

Haematologica 2019

Volume 104(6):1150-1155

\section{Correspondence:}

CATHERINE ROCHE-LESTIENNE catherine.roche@chru-lille.fr

Received: October 8, 2018.

Accepted: December 18, 2018.

Pre-published: December 20, 2018.

doi:10.3324/haematol.2018.208801

Check the online version for the most updated information on this article, online supplements, and information on authorship \& disclosures: www.haematologica.org/content/104/6/1150

\section{(C)2019 Ferrata Storti Foundation}

Material published in Haematologica is covered by copyright. All rights are reserved to the Ferrata Storti Foundation. Use of published material is allowed under the following terms and conditions:

https://creativecommons.org/licenses/by-nc/4.0/legalcode. Copies of published material are allowed for personal or internal use. Sharing published material for non-commercial purposes is subject to the following conditions:

https://creativecommons.org/licenses/by-nc/4.0/legalcode, sect. 3. Reproducing and sharing published material for commercial purposes is not allowed without permission in writing from the publisher.
Audrey Bidet, ${ }^{1}$ Stéphanie Dulucq, ${ }^{1}$ Thomas Smol, ${ }^{2,3}$ Alice Marceau-Renaut, ${ }^{4,5}$ Stéphane Morisset, ${ }^{6}$ Valérie Coiteux, ${ }^{7}$ Marie-Pierre Noël-Walter, ${ }^{7}$ FranckEmmanuel Nicolini, ${ }^{6,8}$ Isabelle Tigaud, ${ }^{9}$ Isabelle Luquet, ${ }^{10}$ Stéphanie Struski, ${ }^{10}$ Baptiste Gaillard, ${ }^{11}$ Dominique Penther,${ }^{12}$ Sylvie Tondeur, ${ }^{13}$ Nathalie Nadal, ${ }^{14}$ Eric Hermet, ${ }^{15}$ Lauren Véronèse,${ }^{16}$ Delphine Réa, ${ }^{17}$ Carine Gervais, ${ }^{18}$ Olivier Theisen, ${ }^{19}$ Christine Terré, ${ }^{20}$ Pascale Cony-Makhoul, ${ }^{21}$ Christine Lefebvre, ${ }^{22}$ Jean-Baptiste Gaillard, ${ }^{23}$ Isabelle Radford ${ }^{24}$ Anne-Laure Vervaeke, ${ }^{1}$ Carole Barin, ${ }^{25}$ Elise Chapiro, ${ }^{26}$ Florence Nguyen-Khac, ${ }^{26}$ Gabriel Etienne, ${ }^{27}$ Claude Preudhomme, ${ }^{3,4,5}$ François Xavier Mahon ${ }^{27}$ and Catherine Roche-Lestienne ${ }^{2,3,5}$ on behalf of the Groupe Francophone de Cytogénétique Hématologique (GFCH) and the French Intergroup of Chronic Myeloid Leukemia (Fi-LMC).

${ }^{1}$ Laboratoire d'Hématologie, CHU Bordeaux; ${ }^{2}$ Institut de Génétique Médicale, Hôpital Jeanne de Flandre, CHU Lille; ${ }^{3}$ Centre de Recherche Jean-Pierre Aubert, UMR-S 1172, Université de Lille; ${ }^{4}$ nnstitut d'Hématologie, Centre de Biologie Pathologie Génétique, CHU Lille; ${ }^{5}$ Inserm, UMR-S 1172, Lille; ' ${ }^{6}$ épartement d'Hématologie, Centre Léon Bérard, Lyon; ${ }^{7}$ Service des Maladies du Sang, Hôpital Claude Huriez, CHU Lille; ${ }^{8}$ Inserm U1052, Centre de Recherche en Cancérologie, Centre Léon Bérard, Lyon; ' 'Laboratoire de Cytogénétique et de Biologie Moléculaire, Service d'Hématologie Biologique - CBPAS, GHS - Hospices Civils de Lyon, Pierre-Bénite Cedex, France; ${ }^{10}$ Laboratoire d'Hématologie, Plateau Technique Hématologie-Oncologie, Institut Universitaire du Cancer de Tolouse Oncopole; ${ }^{11}$ Laboratoire Central d'Hématologie, Hôpital Robert Debré, Reims; ${ }^{12}$ Laboratoire de Génétique Oncologique, Centre de Lutte Contre le Cancer Henri Becquerel, Rouen; ${ }^{13}$ Laboratoire d'Hématologie-Cytogénétique, CHU Saint-Etienne, Hôpital Nord, SaintEtienne Cedex 2; ${ }^{14}$ Laboratoire de Génétique Chromosomique et Moléculaire, Plateau Technique de Biologie, CHU de Dijon; ${ }^{15}$ Service d'Hématologie Clinique, CHU Estaing, Clermont-Ferrand; ${ }^{16}$ Laboratoire de Cytogénétique, CHU Estaing, Clermont-Ferrand;

${ }^{17}$ Service Clinique des Maladies du Sang, Hôpital St Louis, Paris; ${ }^{18}$ Laboratoire Régional de Cytogénétique Hématologique d'Alsace, CHU de Haute Pierre, Strasbourg Cedex; ${ }^{19}$ Laboratoire de Cytogénétique Hématologique, Plateau Technique Hôtel Dieu, Nantes; ${ }^{20}$ Laboratoire de Cytogénétique du Centre Hospitalier Valence, Le Chesnay; ${ }^{21}$ Service d'Hématologie, Centre Hospitalier Annecy-Genevois, Epagny Metz-Tessy; ${ }^{22}$ Unité de Génétique des Hémopathies, Institut de Biologie et Pathologie, CHU Grenoble Alpes, Grenoble Cedex 9; ${ }^{23}$ Unité de Génétique Médicale et Cytogénétique, CHU de Nîmes; ${ }^{24}$ Laboratoire de Cytogénétique, Hôpital Necker - Enfants Malades, Paris; ${ }^{25}$ Laboratoire de Cytogénétique Onco-Hématologie, Hôpital Bretonneau, Tours; ${ }^{26}$ Service d'Hématologie Biologique, Groupe Hospitalier Pitié-Salpêtrière, Assistance Publique des Hôpitaux de Paris et Sorbonne Université, Paris and ${ }^{27}$ Département d'Hématologie, Institut Bergonié, Bordeaux, France.

\section{ABSTRACT}

lonal chromosome abnormalities in Philadelphia-negative cells could concern chronic myeloid leukemia patients treated by tyrosine kinase inhibitors. The European LeukemiaNet distinguishes $-7 / \operatorname{del}(7 q)$ abnormalities as a "warning". However, the impact of clonal chromosome abnormalities, and specifically those of $-7 / \operatorname{del}(7 q)$, in Philadelphia-negative cells on clinical outcomes is unclear and based on case-reports showing morphological dysplasia and increased risk of acute myeloid leukemia, suggesting the coexistence of chronic myeloid leukemia and high-risk myelodysplastic syndrome. The aim of this study was to determine whether the impact of $-7 / \mathrm{del}(7 \mathrm{q})$ clonal chromosome abnormalities in Philadelphia-negative cells on the clinical outcome is different from that of other types of abnormalities, and we argue for an underlying associated high-risk myelodysplastic syndrome. Among 102 chronic myeloid 
leukemia patients with clonal chromosome abnormalities in Philadelphia-negative cells with more than a median of 6 years of follow up, patients with -7/del(7q) more frequently had signs of dysplasia, a lower cumulative incidence of deep molecular response and often needed further treatment lines, with the consequent impact on event-free and progression-free survival. Morphological features of dysplasia are associated with myelodysplastic syndrome/acute myeloid leukemia mutations and compromise the optimal response to tyrosine kinase inhibitors, irrespectively of the type of clonal chromosome abnormalities in Philadelphianegative cells. However, mutation patterns determined by next-generation sequencing could not clearly explain the underlying high-risk disease. We hereby confirm the pejorative prognostic value of $-7 / \mathrm{del}(7 \mathrm{q})$ clonal chromosome abnormalities in Philadelphia-negative cells and suggest that myelodysplastic features constitute a warning signal that response to tyrosine kinase inhibitors may be less than optimal.

\section{Introduction}

Concurrently with the $B C R-A B L 1$ fusion gene resulting from $\mathrm{t}(9 ; 22)(\mathrm{q} 34 ; \mathrm{q} 11)$ in Philadelphia-positive $\left(\mathrm{Ph}^{+}\right)$cells, clonal chromosome abnormalities (CCA) can be present at the time of diagnosis of chronic myeloid leukemia (CML) or emerge during therapy. CCA in $\mathrm{Ph}^{+}$cells $\left(\mathrm{CCA} / \mathrm{Ph}^{+}\right)$are well known; they are associated with clonal cytogenetic evolution and failure of tyrosine kinase inhibitor (TKI) therapy. ${ }^{1}$ However, CCA may also occur in Philadelphia-negative cells (CCA/Ph). According to reported series, CCA/Phcould be present in $2 \%$ to $17 \%$ of CML patients treated with TKI. These differences in frequencies could be partly explained by taking into account (or not) the loss of chromosome $\mathrm{Y}$ and transitory abnormalities. ${ }^{2-7}$ While the frequency of CCA/Ph varies greatly from study to study, other characteristics seem more reproducible, such as the median age at onset (between 49 and 58 years), the median time of the first appearance during TKI therapy (between 10 and 17 months) and the type of CCA/Ph which is, according to their frequencies: trisomy $8(+8)$, monosomy 7/deletion 7q [-7/del(7q)], loss of the Y chromosome (-Y), deletion 20q (20q) and others. Despite very limited information on the occurrence of this phenomenon among patients treated with second-generation TKI, the incidence and type of abnormalities after nilotinib or dasatinib treatment seem to be similar to those reported in patients after imatinib therapy. $3,7,8$

Controversies still exist regarding the emergence of $\mathrm{CCA} / \mathrm{Ph}$, not only on the time of appearance (before or after treatment), but also on the potential impact of the type of TKI or high doses of TKI. For Kovitz et al. ${ }^{9}$ the appearance of CCA was not observed in the Ph cells prior to therapy with interferon or imatinib. This pleads for a supporting action of the treatment or a selective pressure on progenitor cells. Conversely, HUMARA-polymerase chain reaction and deep mutational screening of $\mathrm{Ph}$ cells could argue for the existence of clonal $\mathrm{Ph}$ hematopoiesis, either before or after CML therapy. ${ }^{10,11}$ It could then be speculated that a clonal $\mathrm{Ph}$ state could precede the acquisition of $B C R$ $A B L 1$, as evidence of a two-step model of CML. Another hypothesis is that $\mathrm{Ph}^{+}$or $\mathrm{Ph}$ concomitant clones arise from genetically unstable progenitors, with the $\mathrm{Ph}$ clone revealed at the time of TKI-induced remission of CML.

Most CCA/Ph are similar to those commonly associated with myelodysplastic syndromes (MDS) or secondary acute myeloid leukemia (AML), raising many questions about their association with myelodysplasia and the risk of transformation to MDS or AML. In 2013, the European LeukemiaNet (ELN) recommendations, ${ }^{1}-7 / \operatorname{del}(7 \mathrm{q})$ abnormalities are considered as a "warning" compared to other
$\mathrm{CCA} / \mathrm{Ph}$. This is an innovation compared to the $2009 \mathrm{ELN}$ recommendations. This singling out of $-7 / \mathrm{del}(7 \mathrm{q})$ abnormalities was based on some case reports indicating MDS features and an increased risk of AML development among patients with such abnormalities, not present with other $\mathrm{CCA} / \mathrm{Ph}$ in the absence of dysplasia. ${ }^{2,9,12-14}$ Indeed Deninger et al. reported 17 patients treated with imatinib after failure of interferon therapy who had CCA/Ph and developed MDS; eight of them had -7. ${ }^{2}$ In a meta-analysis, Groves et al. found that $32 \%$ of $16 \mathrm{CCA} / \mathrm{Ph}$ patients with $-7 \mathrm{had}$ transformation to MDS/AML. ${ }^{12}$ However, the prognostic impact of CCA/Ph, and specifically those of chromosome 7 , on clinical outcomes is unclear as only limited studies have had a sufficiently long follow up. ${ }^{4} \mathrm{We}$, therefore, conducted a large, retrospective French multicenter study of CCA/Ph CML patients with a prolonged follow up to: (i) evaluate the frequency of abnormalities of chromosome 7 among CCA/Ph, especially in the era of second-generation TKI; (ii) determine their impact on clinical outcomes; and (iii) assess the existence of an underlying associated highrisk MDS by systematic bone marrow morphological review and next-generation sequencing (NGS) using a MDS/AML panel.

\section{Methods}

\section{Patients}

The databases of French institutions were screened, leading to the identification of $102 \mathrm{CML}$ patients with a CCA/Ph at diagnosis or during the course of treatment with one or more lines of TKI, and $11 \mathrm{MDS}$ patients without a history of CML who had an abnormal karyotype who formed the control group. Among these patients, a chromosome 7 abnormality [-7/del(7q)] was detected in $26 \mathrm{CML}$ cases and in four MDS cases. Patients in blast phase CML at diagnosis were excluded.

The patients' clinical and biological data were obtained from medical records. The study was performed in accordance with the Declaration of Helsinki, and was approved by a local investigational review board (CPP DC 2015/133).

\section{Procedures}

Cytogenetic response was determined using standard procedures. Clonality was defined by two or more metaphases presenting the same abnormality, or three metaphases presenting the same monosomy except for $-Y$ because the rate of this monosomy could be age-related. The potential involvement of $-Y$ in the leukemic process was only established for patients with more than $75 \%$-Y metaphases. All karyotypes were reviewed by Groupe Francophone de Cytogénétique Hématologique members and then classified according to the 2016 International System for Human 
Cytogenetic Nomenclature. Molecular monitoring was performed according to the ELN recommendations. ${ }^{1}$ A morphological central review was used to screen for myelodysplastic features at the time of CCA/Ph emergence in 48 cases. Morphological dysplasia was considered significant when it was observed for $10 \%$ or more cells in any hematopoietic lineage with or without excess of blasts $(>5 \%)$. Erythroid lineage dysplasia criteria include nuclear and cytoplasmic abnormalities (multinuclearity, laminated cytoplasm, macroerythroblasts). Dysgranulopoiesis also includes hypogranular or hypergranular precursors and/or neutrophils and/or lack of nuclear segmentation. Micromegakaryocytes, multinuclear or hypolobulated megakaryocytes were the main abnormalities observed in the megakaryocyte lineage. Patients were stratified according to the presence or absence of chromosome 7 abnormalities, whether isolated or not, leading to $-7 / \mathrm{del}(7 \mathrm{q}) \mathrm{CCA} / \mathrm{Ph}$ identified by conventional cytogenetics. In the case of $\mathrm{CCA} / \mathrm{Ph}$ detection after the diagnosis of CML, time of emergence was retrospectively evaluated on prior samples by fluorescence in situ hybridization when possible. Complex karyotypes ( $\geq 3$ anomalies) affected only five of the 102 patients; since this precluded statistically meaningful analyses, these abnormalities were not considered as a separate category. Underlying MDS was documented both by centralized morphological analysis of bone marrow smears and by NGS for a targeted panel of 27 genes frequently altered in MDS and AML (ASXL1, CBL, CEBPA, DNMT3A, ETV6, EZH2, FLT3, IDH1, IDH2, JAK2, KIT, KRAS, MPL, NPM1, NRAS, PHF6, PTPN11, RIT1, RUNX1, SETBP1, SF3B1, SRSF2, TET2, TP53, U2AF1, WT1, ZRSR2). NGS data obtained for $45 \mathrm{CML}$ patients were compared with a MDS control group of patients with $(n=4)$ or without ( $\mathrm{n}=7$ ) cytogenetic chromosome 7 anomalies. NGS was performed as previously described ${ }^{15}$ on follow-up bone marrow samples at complete cytogenetic response and preferably at best molecular response, to limit the risk of residual $\mathrm{Ph}^{+}$clone contamination.

The quality of response to treatment was classified by the best cytogenetic or molecular response obtained at any point after one, two, three or more lines of treatments into no cytogenetic response, partial cytogenetic response, complete cytogenetic response, major molecular response and deep molecular response $\leq 0.0032 \%$ (MR4.5). The response criteria were according to the standard ELN definitions. ${ }^{1}$

\section{Statistical analysis}

Bivariate analyses were performed to compare $-7 / \operatorname{del}(7 \mathrm{q}$ versus other CCA/Ph: Quantitative variables were described by their mean and standard deviation in both groups and compared by a Student $t$ test if the distribution was normal or with their median, range and quartiles along with a non-parametric Mann-Whitney test when the distribution was not normal. Cumulative incidences from diagnosis or initiation of first-line TKI treatment until the achievement of the considered response were calculated with death without response as a competing risk. Gray tests were performed to compare cumulative incidence curves. Analyses from time of CCA/Ph emergence were not performed because detection time may be delayed and underestimated for some patients depending on the clinical context and requirement of additional cytogenetic controls despite complete cytogenetic response. However, the effect of type of CCA/Ph on response, progression, or late events was considered by landmark analyses 3 years after the initiation of TKI therapy. Probabilities of overall survival, progression-free survival and event-free survival since initiation of first-line TKI therapy were calculated and illustrated by the Kaplan-Meier method: until death at any time and for any reason (overall survival); until death or progression to accelerated phase or blastic transformation (progression-free survival); and until intoler- ance, loss of response, resistance, treatment switch, progression, or death (event-free survival). Survival curve comparisons, including landmark analyses, were performed using log-rank tests. The level of statistical significance was set at $5 \%$. All the statistical analyses were performed with R V3.2.3.

\section{Results}

\section{Clinical and cytogenetic presentation of patients with chronic myeloid leukemia}

The patients' baseline characteristics are summarized in Online Supplementary Table S1). Twenty-six out of the 102 $(25.5 \%)$ patients had CCA/Ph affecting chromosome 7 , which was an isolated abnormality in 13 out of the 26 cases $(50 \%)$ and associated with +8 in $19.5 \%$ cases. In one patient with $-7 \mathrm{CCA} / \mathrm{Ph}$, +8 was detected in a separate clone. Among other $\mathrm{CCA} / \mathrm{Ph}$ cases, +8 was the most frequent other abnormality, affecting 31 out of the 46 patients $(67.4 \%) ; 13.7 \%(\mathrm{n}=14)$ patients had -Y CCA/Ph. Patients who developed $-7 / \operatorname{del}(7 q)$ CCA/Ph were significantly younger (mean 48 vs. 55 years old; $P=0.035$ ) and mostly benefited from second-generation TKI as first-line treatment (mainly dasatinib: $15.4 \%$ vs. $2.6 \%$ cases in the other $\mathrm{CCA} / \mathrm{Ph}$ group; $P=0.027$ ). Other baseline characteristics were similar between the $-7 / \mathrm{del}(7 \mathrm{q}) \mathrm{CCA} / \mathrm{Ph}$ cases and the other $\mathrm{CCA} / \mathrm{Ph}$ cases. The median follow up from the diagnosis of CML was 6.47 (range, 1-19) years. The median time of CCA/Ph detection after starting TKI therapy was 2.08 (range, -0.8 to 12.65 ) years for $-7 / \mathrm{del}(7 \mathrm{q}) \mathrm{CCA} / \mathrm{Ph}$ and 1.02 (range, -3.32 to 11.02 ) years for other CCA/Ph: The -7 $\mathrm{CCA} / \mathrm{Ph}$ was present at diagnosis in only one case, as determined by retrospective fluorescence in situ hybridization analysis. Twelve out of the 26 patients who developed $7 / \mathrm{del}(7 \mathrm{q}) \mathrm{CCA} / \mathrm{Ph}$ were in complete cytogenetic response at the time the abnormality was detected. The median follow up since CCA/Ph detection was 5.35 (range, 1-14) years for $-7 / \operatorname{del}(7 q)$ and 7.41 (range, $0-15$ ) years for other $\mathrm{CCA} / \mathrm{Ph}(P \geq 0.05)$. The trend approached statistical significance for the number of treatment lines required after the first 3 years of follow up, as patients with $-7 / \operatorname{del}(7 q)$ $\mathrm{CCA} / \mathrm{Ph}$ more frequently needed two or more treatment lines $(30 \%$ vs. $13.10 \%$ of patients with other CCA/Ph; $P=0.049$, chi-square).

\section{Biological analysis of underlying associated myelodysplastic syndrome}

The presence of MDS features at the time of CCA/Ph; determined by morphological analysis of the bone marrow, was evaluated in 48 patients and appeared to alter the quality of response to treatment, as only $9.4 \%$ of patients with MDS signs (24 patients) reached a major molecular response or better versus $47 \%$ of patients without $\mathrm{MDS}$ signs $(P<0.0001$, chi-square). Morphological MDS features were more frequent among the $-7 /$ del7 $q$ CCA/Ph group $[50 \%$ of patients with $-7 / \operatorname{del}(7 \mathrm{q}) \mathrm{CCA} / \mathrm{Ph}$ vs. $20 \%$ with other CCA/Ph], and only $33 \%$ (4 out of 12 ) of the $-7 / \operatorname{del}(7 q)$ patients with $M D S$ signs achieved a major molecular response or better compared to $75 \%$ ( 9 out of 12) of these patients without MDS signs. For patients with other types of CCA/Ph; only six out of 49 patients $(18.8 \%)$ with an optimal response had morphological features of MDS $(P<0.00001$, chi-square). NGS was performed for $45 \mathrm{CML}$ patients with available DNA at the time of detection of $\mathrm{CCA} / \mathrm{Ph}^{-}$and $11 \mathrm{MDS}$ patients as a 


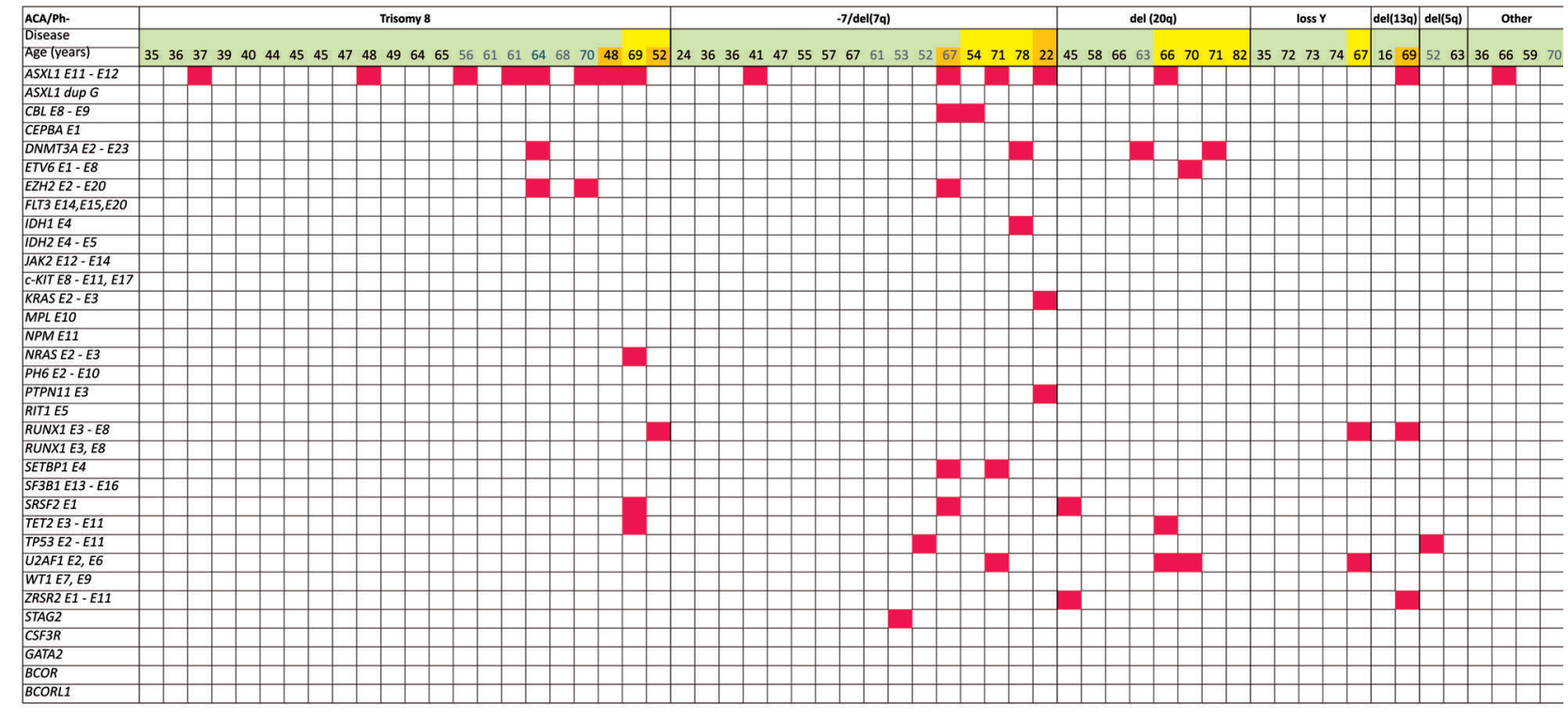

Figure 1. Landscape of mutations detected by next-generation sequencing in patients with chronic myeloid leukemia and myelodysplastic syndromes. Columns (patients) are first sorted by the type of clonal chromosome abnormalities in Philadelphia chromosome-negative cells (CCA/Ph), then by disease [chronic myeloid leukemia in green, myelodysplastic syndrome (MDS) in yellow, advanced disease in orange) and by the presence of morphological features of MDS (in blue characters) from the youngest to the oldest. Pink cells indicate somatic mutations that were detected.

control group (Figure 1). At least one mutation was identified in $90 \%$ (10 out of 11 patients) of the MDS group versus $37 \%$ (16 out of 43 patients) of the CCA/Ph CML group. The proportion of patients with mutations in the MDS group was consistent with that reported in the literature ${ }^{16}$ and the MDS/AML mutations detected were significantly associated with the MDS group versus $\mathrm{CCA} / \mathrm{Ph}$ CML patients $(P<0.0011$, chi-square). In CML cases, 13 out of the 24 patients with morphological signs of MDS had DNA available for NGS. No significant association was found between NGS-detected mutations and patients' age or type of CCA/Ph but MDS morphological features were significantly associated with the presence of mutations as nine $(68.75 \%)$ of the patients with morphological signs of MDS had mutations versus four $(31.25 \%)$ without signs $(P<0.00001$, chi-square). This result supports the presence of an underlying MDS disease in CML patients with observed morphological signs of dysplasia. Among the 12 patients with -7/del(7q), four had morphological signs of MDS and three had mutations. Two of them had some mutations in a gene known to be implicated in disease progression: one patient had an undescribed EZH2 c.826C>T:p.Gln276* alteration with a low variant frequency $(1 \%)$, and the other had an isolated TP53 c.709A >C:p.Met273Leu mutation (variant frequency, $71 \%$ ) but reached MR4.5 after first-line TKI treatment with dasatinib. The other two have an isolated mutation of ASXL1 or STAG2. Of note, EZH2 on chromosome 7 was also mutated in two other patients with $+8 \mathrm{CCA} / \mathrm{Ph}$ : one with EZH2 c.2079T>A: p.Asn693Lys (variant frequency, $17 \%$ ) with a poor response to second-line TKI and the other with EZH2 c.826C>T:p.GIn276* (variant frequency, $1 \%$ ) with a poor response after third-line TKI.

\section{Outcomes and survival}

The cumulative incidence of MR4.5 is illustrated in Figure 2. The median time to MR4.5 after starting TKI therapy
A

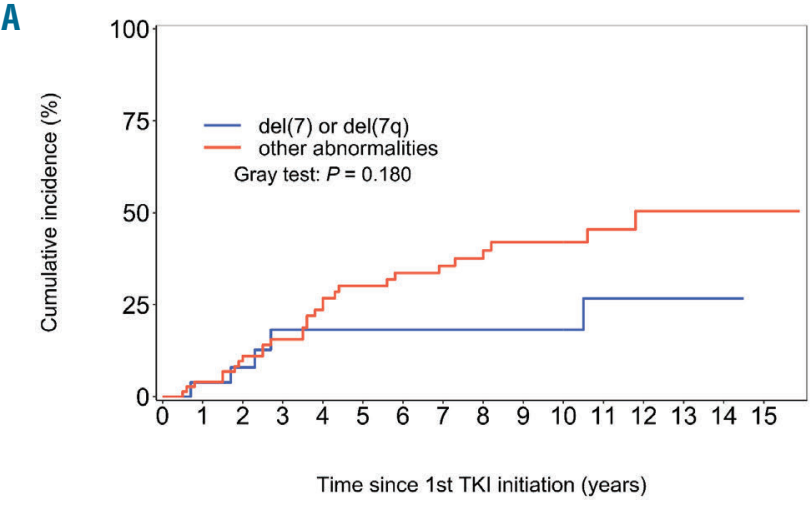

B

Cumulative incidence of MR4.5

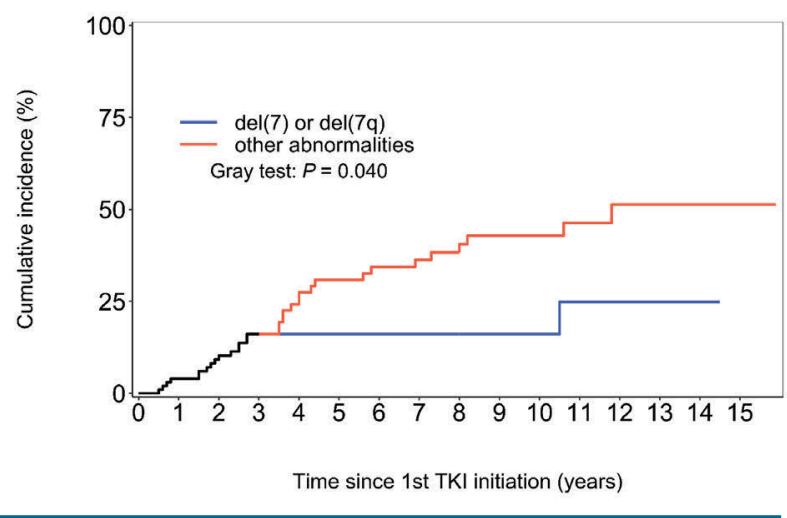

Figure 2. Cumulative incidence of deep molecular response following the first line of tyrosine kinase inhibitor therapy. (A) Cumulative incidence of deep molecular response (MR4.5) following initiation of the first line of tyrosine kinase inhibitor (TKI) therapy according to the type of clonal chromosome abnormalities in Philadelphia chromosome-negative cells (CCA/Ph). (B) Cumulative incidence of MR4.5 according to the type of CCA/Ph by landmark analysis 36 months after initiation of the first line of TKI therapy. 
was 2.3 (range, $0.66-10.5$ ) years for $-7 / \mathrm{del}(7 \mathrm{q}) \mathrm{CCA} / \mathrm{Ph}$ patients versus 3.55 (range, $0.5-11.77$ ) years for patients with other $\mathrm{CCA} / \mathrm{Ph}-(P=\mathrm{NS}$, exact Mann-Whitney test) (Figure 2A). However, landmark analysis after 3 years of therapy revealed the adverse effect of $-7 / \mathrm{del}(7 \mathrm{q}) \mathrm{CCA} / \mathrm{Ph}(P=0.04)$ (Figure 2B) on the cumulative incidence of MR4.5. The type of CCA $/ \mathrm{Ph}$ did not have an impact on overall survival $(P=0.717$; data not shown). The overall rates of progressionfree survival at 3,5 and 10 years were $94.74 \%$ [95\% confidence interval (95\% CI): 90.35-99.35], 86.28\% (95\% CI: 78.94-94.30), and $78.60 \%$ (95\% CI: 69.12-89.39), respectively. The overall rates of event-free survival at 3,5 and 10 years were $67.36 \%$ (95\% CI: 58.63-77.39), 56.32\% (95\% CI: $46.72-67.90$ ), and $43.61 \%$ (95\% CI: $33.34-57.06$ ), respectively (data not shown). Landmark analyses at 3 years after starting first TKI therapy revealed a strong negative impact of $-7 / \mathrm{del}(7 \mathrm{q}) \mathrm{CCA} / \mathrm{Ph}$ on both event-free and progression-free survival $(P=0.015$ and $P=0.022$, respectively, log-rank test), as shown in Figure 3.

\section{Discussion}

To our knowledge, this is the largest study of CML patients with CCA/Ph, with $26(25.4 \%)$ out of 102 patients having $-7 / \mathrm{del}(7 \mathrm{q})$ abnormalities in a consistent, long follow up [median 6.47 (range, 1-11) years]. Our cohort required particular care as $37 \%$ of them needed at least two switches of TKI prior to achieving their optimal response. Patients who developed $-7 / \operatorname{del}(7 \mathrm{q}) \mathrm{CCA} / \mathrm{Ph}$ were significantly younger (means 48 years old) and mostly benefited from second- and/or third-generation TKI, with a significantly lower cumulative incidence of MR4.5 at the landmark analysis 3 years after starting TKI therapy $(P=0.04)$ than other patients. Almost half of the patients (46\%) with 7/del(7q) CCA $/ \mathrm{Ph}^{-}$were in complete cytogenetic remission at the time of detection of the CCA/Ph. Because the quality of cytogenetic responses significantly affects the prognosis, the high proportion of good cytogenetic responders in this group strengthens the involvement of chromosome 7 abnormalities in our prognostic results. Furthermore, additional cytogenetic abnormalities in $\mathrm{Ph}^{+}$cells (which are considered as clonal evolution with greater risk of transformation) cannot be suspected to be involved in prognosis in our study because only one out of 102 patients had an atypical $\mathrm{t}(7 ; 14)(\mathrm{p} 21 ; \mathrm{q} 12)$ translocation in $\mathrm{Ph}^{+}$cells at diagnosis.

Associated dysplastic features, identified by morphological analysis of bone marrow, seemed to be a more frequent event in the $-7 / \operatorname{del}(7 q)$ group, and had a significant negative impact on quality of response and outcomes as compared to that in other types of CCA/Ph cases. In addition, irrespectively of the type of CCA/Ph; patients with morphological MDS signs had significantly lower rates of major molecular response $(P<0.0001)$. Likewise, $78 \%$ of the patients who did not have a complete cytogenetic response were those who had morphological features of MDS. This is in accordance with the findings of a preceding study by Deininger et al. ${ }^{2}$ who reported that patients with isolated CCA/Ph without morphological evidence of dysplasia do not require special management. In a study by Terré et al., CCA/Ph were not associated with myelodysplasia and did not impair the cytogenetic response to imatinib. ${ }^{6}$ However, although this was the largest published series of $\mathrm{CCA} / \mathrm{Ph}$. cases to date, only two out of 28 evaluated patients had dysgranulopoiesis, the median follow up was short (only 24
A

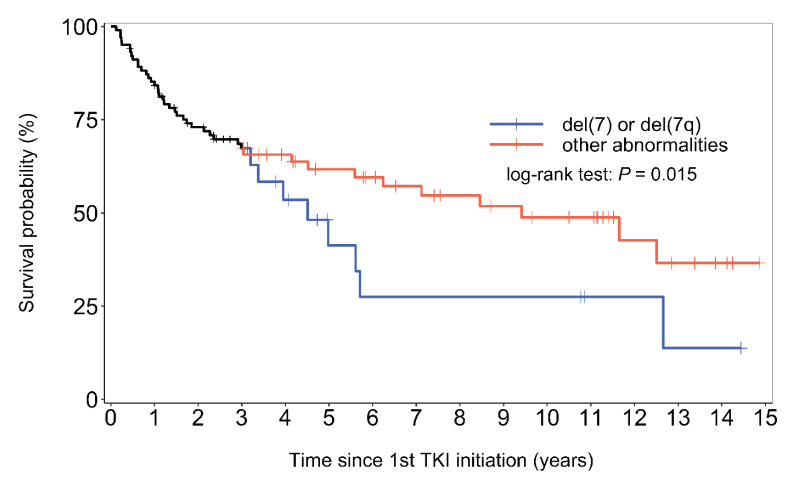

B

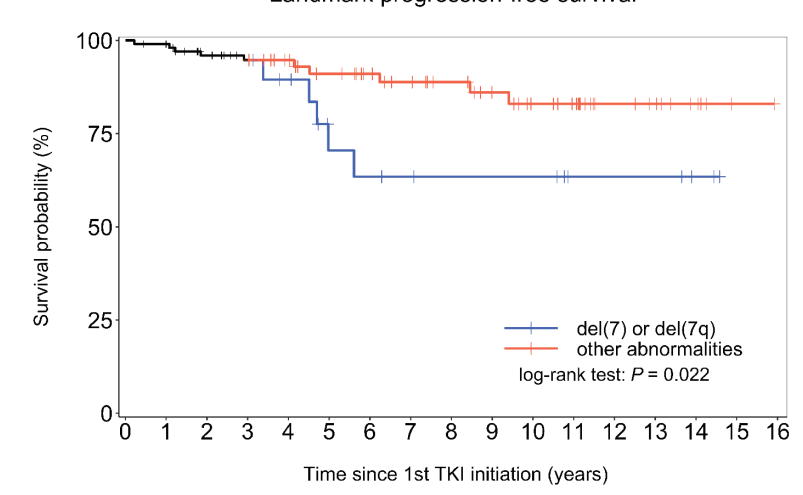

Figure 3. Landmark survival analyses according to the type of clonal chromosome abnormalities in Philadelphia chromosome-negative cells 36 months after initiation of the first line of tyrosine kinase inhibitor therapy. (A) Eventfree survival. (B) Progression-free survival. TKI: tyrosine kinase inhibitor.

months after imatinib initiation), and the majority of patients in this study had been previously treated.

Even though the overall survival rates were not statistically significantly different between the two groups, landmark analyses of the impact of $-7 / \mathrm{del}(7 \mathrm{q}) \mathrm{CCA} / \mathrm{Ph}$ after 3 years of starting TKI treatment revealed lower event-free and progression-free survival rates $(P=0.015$ and $P=0.022$, respectively). Recently, Issa et al. ${ }^{4}$ reported the prognostic relevance of CCA/Ph in 58 patients with a median follow-up of 7.6 years. Among them, only four patients had $-7 / \operatorname{del}(7 q)$ type abnormalities. The disease progressed in three of these four patients, transforming to either MDS or CML blast crisis, without an obvious link with MDS signs in this small cohort. Excluding -Y CCA/Ph; a decreased survival was observed for patients with $\mathrm{CCA} / \mathrm{Ph}$ and seemed to be related to MDS or CML transformation. A specific -7/del(7q)associated risk could not be determined in their study. In another recent study, Wasilewska et al. found that -7 and +8 CCA/Ph did not have an impact on the long-term outcome of CML patients, but survival was not investigated in this study and the series was limited to five observations. ${ }^{17}$

The occurrence of $\mathrm{Ph}^{+}$and $\mathrm{CCA} / \mathrm{Ph}$ in the same patient could suggest the presence of two concurrent, distinct hematologic disorders: CML and MDS. To investigate the biological features of such underlying diseases, we screened for mutations in frequently altered genes in MDS or AML by NGS at the time of best response to therapy. Mutations were detected more frequently in our MDS control group of patients than in the CML group, and were also mostly found 
in CCA/Ph CML patients with features of MDS ( $P=0.006)$. Mutations were not associated with age and there was no preferentially significant association with $-7 / \operatorname{del}(7 q)$, despite a higher rate of $\mathrm{MDS}$ features in this subgroup. As already reported in CML, ${ }^{18-20}$ ASXL1 is one of the most frequently mutated genes. Although somatic mutation or decreased expression of EZH2 at 7q36.1 plays a role in cancer, the molecular mechanisms responsible for the poor prognosis of chromosome 7 alterations in hematologic diseases remains undefined. In this study, three patients had EZH2 mutations. One of them had -7 CCA/Ph; thus suggesting a bi-allelic alteration. This patient further progressed to an advanced phase of CML, but the direct role of the EZH2 mutation in this case could not be determined because the frequency of the variant was low $(1 \%)$ and it was associated with other mutations in ASXL1, CBL, SETBP1 and SRSF2. The two other patients with $E Z H 2$ mutations had +8 CCA/Ph; associated in one case with an ASLX1 mutation, and neither of them had further disease evolution. It has been described that NGS screening of CML patients in major molecular response revealed a higher rate of other gene mutations in patients with CCA/Ph than in patients without CCA/Ph ${ }^{-21}$ These latter mutational events may therefore be independent, and could result from genetic instability.

In conclusion, in this study we were able to demonstrate the negative prognostic impact of $-7 / \mathrm{del}(7 \mathrm{q}) \mathrm{CCA} / \mathrm{Ph}$ on the cumulative incidences of MR4.5, event-free survival and progression-free survival in a cohort of $102 \mathrm{CML}$ patients with $\mathrm{CCA} / \mathrm{Ph}$ with a median follow up of more than 6 years. Morphological features of MDS represent a negative prognostic factor for optimal response to treatment, irrespectively of the type of CCA/Ph, even though they are more frequent in the $-7 / \operatorname{del}(7 q)$ CCA/Ph subgroup. NGS screening for $\mathrm{MDS} / \mathrm{AML}$ mutations failed to clearly explain the underlying high risk of myelodysplastic disease in the $-7 / \mathrm{del}(7 \mathrm{q}) \mathrm{CCA} / \mathrm{Ph}$ group, but the presence of mutations in genes of our MDS/AML panel was statistically associated with morphological features of MDS on bone marrow smears. Further analyses will help to determine whether morphological signs of MDS in the bone marrow and/or MDS mutations at the time of emergence of $\mathrm{CCA} / \mathrm{Ph}$ represent a warning signal in these particular CML patients.

\section{Funding}

This work was funded with the support of the Fi-LMC group, which received partial contributions from Bristol Myers Squibb, Novartis and Incyte Pharma.

\section{Acknowledgments}

The authors would like to thank Laurence Meyer for her morphological bone marrow partial review, and Olivier Nibourel, Jean-Michel Cayuela, and Sandrine Hayette for their BCR-ABL1 molecular monitoring data.

\section{References}

1. Baccarani M, Deininger MW, Rosti G, et al. European LeukemiaNet recommendations for the management of chronic myeloid leukemia: 2013. Blood. 2013;122(6):872884.

2. Deininger MWN, Cortes J, Paquette R, et al. The prognosis for patients with chronic myeloid leukemia who have clonal cytogenetic abnormalities in Philadelphia chromosome-negative cells. Cancer. 2007;110(7): 1509-1519.

3. De Melo VAS, Milojkovic D, Khorashad JS, et al. Philadelphia-negative clonal hematopoiesis is a significant feature of dasatinib therapy for chronic myeloid leukemia. Blood. 2007;110(8):3086-3087.

4. Issa GC, Kantarjian HM, Gonzalez GN, et al. Clonal chromosomal abnormalities appearing in Philadelphia chromosomenegative metaphases during CML treatment. Blood. 2017;130(19):2084-2091

5. Lee S-E, Choi SY, Bang J-H, et al. The longterm clinical implications of clonal chromosomal abnormalities in newly diagnosed chronic phase chronic myeloid leukemia patients treated with imatinib mesylate. Cancer Genet. 2012;205(11):563-571.

6. Terre C, Eclache V, Rousselot P, et al. Report of 34 patients with clonal chromosomal abnormalities in Philadelphia-negative cells during imatinib treatment of Philadelphia-positive chronic myeloid leukemia. Leukemia. 2004;18(8):1340 1346.

7. Wang $\mathrm{H}$, Jin J, Wang $Y$, Huang $X$, Huang J. Clonal chromosomal abnormalities in Philadelphia-negative cells in chronic myeloid leukemia patients treated with nilotinib used in first-line therapy. Ann Hematol. 2013;92(12):1625-1632

8. Baldazzi C, Luatti S, Marzocchi G, et al. Emergence of clonal chromosomal abnormalities in Philadelphia negative hematopoiesis in chronic myeloid leukemia patients treated with nilotinib after failure of imatinib therapy. Leuk Res. 2009;33 (12):e218-220.

9. Kovitz C, Kantarjian H, Garcia-Manero G, Abruzzo LV, Cortes J. Myelodysplastic syndromes and acute leukemia developing after imatinib mesylate therapy for chronic myeloid leukemia. Blood. 2006;108(8): 2811-2813.

10. Paquette RL, Nicoll J, Chalukya M, et al. Clonal hematopoiesis in Philadelphia chromosome-negative bone marrow cells of chronic myeloid leukemia patients receiving dasatinib. Leuk Res. 2010;34(6):708713 .

11. Schmidt M, Rinke J, Schäfer V, et al. Molecular-defined clonal evolution in patients with chronic myeloid leukemia independent of the BCR-ABL status. Leukemia. 2014;28(12):2292-2299.

12. Groves MJ, Sales M, Baker L, Griffiths M, Pratt N, Tauro S. Factors influencing a second myeloid malignancy in patients with Philadelphia-negative -7 or $\operatorname{del}(7 q)$ clones during tyrosine kinase inhibitor therapy for chronic myeloid leukemia. Cancer Genet. 2011;204(1):39-44.

13. Jabbour E, Cortes JE, Kantarjian HM. Suboptimal response to or failure of imatinib treatment for chronic myeloid leukemia: what is the optimal strategy? Mayo Clin Proc. 2009;84(2):161-169.

14. Zeidan A, Kakati S, Anderson B, Barcos M, Wetzler M. Monosomy 7 in $t(9 ; 22)$-negative cells during nilotinib therapy in an imatinib-resistant chronic myeloid leukemia case. Cancer Genet Cytogenet. 2007;176(2):169-171.

15. Renneville A, Attias P. Thomas X, et al. Genetic analysis of therapy-related myeloid neoplasms occurring after intensive treatment for acute promyelocytic leukemia. Leukemia. 2018;32(9):2066-2069.

16. Papaemmanuil E, Gerstung M, Malcovati $\mathrm{L}$, et al. Clinical and biological implications of driver mutations in myelodysplastic syndromes. Blood. 2013;122(22):36163627; quiz 3699

17. Wasilewska EM, Panasiuk B, Gniot M, et al. Clonal chromosomal aberrations in Philadelphia negative cells such as monosomy 7 and trisomy 8 may persist for years with no impact on the long term outcome in patients with chronic myeloid leukemia. Cancer Genet. 2017;216-217:1-9.

18. Boultwood J, Perry J, Zaman R, et al. Highdensity single nucleotide polymorphism array analysis and ASXL1 gene mutation screening in chronic myeloid leukemia during disease progression. Leukemia. 2010;24(6):1139-1145.

19. Roche-Lestienne C, Marceau A, Labis E, et al. Mutation analysis of TET2, IDH1, IDH2 and ASXL1 in chronic myeloid leukemia. Leukemia. 2011;25(10):1661-1664

20. Togasaki E, Takeda J, Yoshida K, et al. Frequent somatic mutations in epigenetic regulators in newly diagnosed chronic myeloid leukemia. Blood Cancer J. 2017;7 (4):e559.

21. Schnittger S, Meggendorfer M, Nadarajah $\mathrm{N}$, et al. In CML patients with good response to TKIs other gene mutations are frequently $(37 \%)$ present in addition to Philadelphia negative, cytogenetically aberrant clones but are rare $(4 \%)$ in cases with MMR and normal karyotype. Blood. 2014;124(21):3126. 